\title{
Aplicación de las metodologías 8D y AMFE para reducir fallos en una fábrica de refrigeradoras
}

JaVIER GABINO IZAGUIRRE NeIRA ${ }^{1}$ María del Rosario PÁrraga Velásquez ${ }^{2}$

RECIBIDO: 11/01/2017 ACEPTADO: 18/05/2017

\begin{abstract}
RESUMEN
En una empresa manufacturera de electrodomésticos de línea blanca se tiene identificado que una línea especifica de refrigeradoras tiene un alto porcentaje de fallos en usuarios finales con respecto a las otras líneas de la misma fábrica. Mediante el análisis de la información que proviene de los servicios técnicos y apoyados en herramientas de calidad, se identifican los principales problemas, se buscan las causas, se proponen mejoras y se previene posteriores fallos. Diversas acciones derivarán a la reducción de problemas en el mercado, colaborará a reducir los costes de garantía de cada producto y sobre todo repercutirá de manera muy favorable para la imagen de la marca.
\end{abstract}

APPLICATION OF QUALITY TOOLS IN A REFRIGERATOR FACTORY IN ORDER TO REDUCE FAILURES IN THE FINAL PRODUCT

\section{ABSTRACT}

In a manufacturing company of white goods, it has identified a specific line of refrigerators that has a high percentage of failures at end users compared with the other lines of the same factory. By analyzing the information coming from the technical services and supported on quality tools the main problems are identified, causes are searched, improvements are proposed and subsequent failures are forewarned. Various actions will result to the reduction of market problems, it will help to reduce warranty costs for each product and especially these will impact very favorable for brand image.

\section{INTRODUCCIÓN}

Según Aldana y Vargas (2011), no existe calidad sin servicio, ni servicio sin calidad, son dimensiones que siempre están presentes de manera articulada para el beneficio y satisfacción de las necesidades demandadas por el hombre. Particularmente, para las empresas comprometidas con la satisfacción del cliente, la información de las quejas más comunes de los clientes es fundamental para la toma de decisiones de la alta dirección ya sea sobre los procesos, los proveedores o diseño del producto. Justamente es aquí donde nace la propuesta que se explicará, debido a que existen diversas oportunidades de mejora en una línea de refrigeradoras de una empresa metalmecánica; se priorizará y se trabajará para una solución robusta ayudados en las herramientas de calidad.

\section{SITUACIÓN PROBLEMÁTICA}

Se cuenta con la información que reportan los servicios técnicos a la empresa en estudio y que están distribuidos en todo el Perú. Esta información está consolidada en una base de datos y se tiene identificado que un porcentaje relevante del total de problemas en mercado en una línea de refrigeradoras se debe al fallo de componentes específicos. Por todo lo anterior es necesario el análisis para encontrar las causas de estos problemas principales y planteamiento de acciones para mejorar el producto final.

\section{FORMULACIÓN DEL PROBLEMA}

¿Se podrá reducir los fallos recurrentes en período de garantía del producto de una línea de refrigeradoras en usuarios finales con el uso de herramientas de calidad?

\section{OBJETIVO}

Identificar y proponer soluciones para reducir los fallos recurrentes en el período de garantía del producto de una línea de refrigeradoras en usuarios finales utilizando las herramientas básicas de calidad y las metodologías 8D y AMFE.

Ingeniero Industrial. Asistente de Calidad de Mercado en BSH Electrodomésticos.Javier Izaguirre@bshg.com

2 Magister en Ingeniería Industrial. Docente Asociada de la UNMSM. mparragav@unmsm.edu.pe. 


\section{MARCO TÉORICO}

\subsection{Metodología 8D}

Según Bosch Group (2013), la metodología 8D es usada para identificar y corregir problemas que se dan con más asiduidad en las empresas; siendo de gran utilidad en la mejora de productos y procesos. Establece una práctica estándar basada en hechos y se concentra en el origen del problema mediante la determinación de la causa raíz.

En el caso de empresas manufactureras, varios problemas suelen presentar síntomas luego de un tiempo de haber pasado por la línea de producción; justamente el 8D recupera la información y establece medidas a corto plazo (no permanentes) para que se permita la producción sin embargo se exige medidas más sólidas para su implementación permanente en línea. Se presentan los 8 pasos de la metodología que se aplicarán directamente a la presente situación problemática:

\section{D1: Formar un equipo}

\section{D2: Definir el problema}

D3: Implementar acciones de contención

D4: Identificar y verificar la causa raíz

D5: Determinar acciones correctivas permanentes

D6: Implementar y verificar las acciones correctivas permanentes

\section{D7: Prevenir la re-ocurrencia del problema y/o su causa raíz}

\section{D8: Reconocer los esfuerzos del equipo}

Para graficar los pasos de la metodología 8D y compararlo con otras metodologías, se muestra en la figura 1 la relación con los procedimientos de probada eficacia para la resolución de problemas como PDCA, Six Sigma y Toyota Business Practices (TBP); aquí se observa que todas estas metodologías se pueden presentar en forma de una estructura común. Se muestra que las secuencias de tareas básicas son las mismas, independientemente del procedimiento o metodología.

\subsection{Metodología AMFE}

Según Méndez (2008), un paso crucial para que el producto sea confiable sería hacer preguntas en la fase de desarrollo como: ¿Qué podría fallar con esta pieza? ¿Qué tan probable es que falle este componente? ¿Qué tan severo sería el fallo de esta pieza para el cliente? El análisis de efectos y modos de falla es una de las técnicas más ampliamente utilizadas para enumerar los posibles modos mediante los cuales los componentes puedan fallar. Se tienen cinco tipos de AMFE: Sistema, diseño, proceso, servicio y software. En el presente estudio se aplicará el AMFE de diseño debido a las características del problema presentado. Aquí los beneficios generales del uso de esta metodología:

- Minimiza el costo de desarrollo y producción.

- Reduce el consumo de tiempo para corregir fallas.

- Captura el conocimiento de la organización.

- Acentúa la prevención del problema.

- Mejora la calidad y confiabilidad del producto y/o proceso.

Se recomienda iniciar el AMFE en etapas muy tempranas del desarrollo del producto como la conceptualización del diseño; esta continuará a través de la vida del producto o servicio. En el procedimiento AMFE, las fallas son priorizadas de acuerdo a qué tan serias son sus consecuencias, qué tan frecuentes ocurren y qué tan fácilmente pueden ser detectadas. Se debe tener en cuenta que el AMFE es un documento vivo que requerirá de su revisión al menos una vez cada año.

Por cada modo de falla el AMFE usa:

- La severidad de la falla si esta ocurriera, ejemplo: impacto en el siguiente proceso, cliente, etc.

- La probabilidad de que este modo de falla ocurra.

- La medición en sitio para detectar cualquier falla antes que hubiera un impacto, ejemplo: impactó en el siguiente proceso, cliente, etc.

- En la figura 2 se aprecia el formato en el cual se debe trabajar para obtener un documento de fácil seguimiento y revisión. Además, en la figura 3 se muestra las valoraciones AMFE para tener una referencia clara de la puntuación necesaria en los campos de severidad, ocurrencia y detección.

\section{METODOLOGÍA}

La metodología a seguir para el uso de herramientas de calidad que nos ayuden a mejorar el producto final se detalla en el siguiente diagrama de flujo (ver figura 4). 


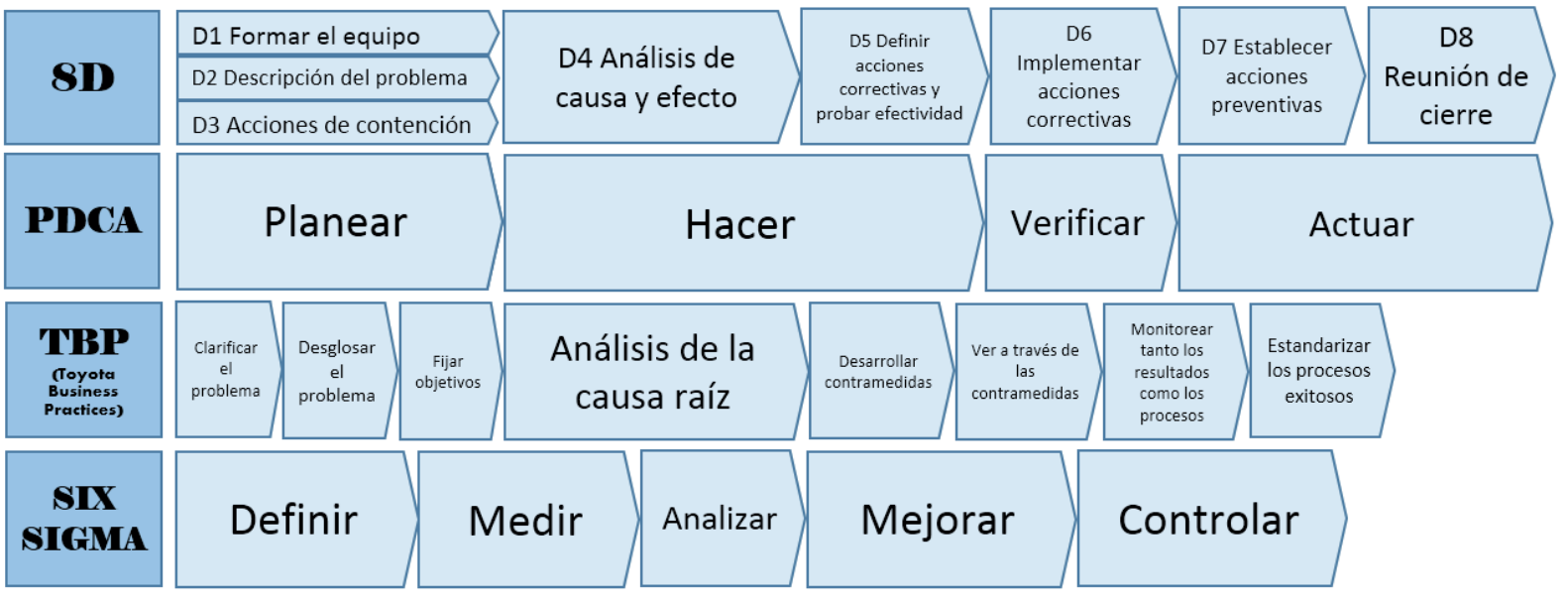

Figura 1. Comparación de las diferentes metodologías para la solución de problemas

Fuente. Empresa en estudio

\begin{tabular}{|c|c|c|c|c|c|c|c|c|c|c|c|c|c|}
\hline No. & $\begin{array}{c}\text { Interface I } \\
\text { Parte I } \\
\text { Proceso }\end{array}$ & Función & $\begin{array}{l}\text { Potencial } \\
\text { Efecto de } \\
\text { Falla }\end{array}$ & 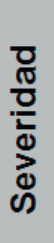 & $\begin{array}{l}\text { Potencial } \\
\text { Modo de } \\
\text { Falla }\end{array}$ & $\begin{array}{c}\text { Potencial } \\
\text { Causa de } \\
\text { Falla }\end{array}$ & $\begin{array}{l}\frac{\pi}{0} \\
\frac{1}{0} \\
0 \\
0 \\
0 \\
0\end{array}$ & $\begin{array}{l}\frac{7}{\pi} \\
\frac{\pi}{0} \\
\frac{0}{0} \\
\frac{ \pm}{2} \\
\end{array}$ & $\begin{array}{l}\text { Modo de } \\
\text { Detección }\end{array}$ & 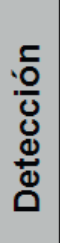 & RPN & $\begin{array}{l}\text { Acciones } \\
\text { sugeridas }\end{array}$ & 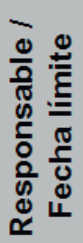 \\
\hline 1 & & & & & & & & & & & & & \\
\hline 2 & & & & & & & & & & & & & \\
\hline 3 & & & & & & & & & & & & & \\
\hline 4 & & & & & & & & & & & & & \\
\hline 5 & & & & & & & & & & & & & \\
\hline
\end{tabular}

Figura 2. Formato de AMFE

Fuente. Empresa en estudio

\begin{tabular}{|c|c|c|c|c|c|}
\hline \multicolumn{2}{|c|}{$\begin{array}{c}\text { S (Severidad) } \\
\text { Cuan severo es el efecto de este tipo de defecto en el } \\
\text { respectivo cliente }\end{array}$} & \multicolumn{2}{|c|}{\begin{tabular}{|c|} 
O (Probabilidad de Ocurrencia) \\
Cuan probable es que el tipo de defecto \\
examinado ocurra en el cliente
\end{tabular}} & \multicolumn{2}{|c|}{$\begin{array}{l}\text { D (Probabilidad de no ser detectado) } \\
\text { Cuan probable es que el defecto llegue al cliente }\end{array}$} \\
\hline CRITERIO & PUNTUACIÓN & CRITERIO & PUNTUACIÓN & CRITERIO & PUNTUACIÓN \\
\hline $\begin{array}{l}\text { Efecto de falla muy grave } \\
\text { - La falla puede provocar situaciones de peligro } \\
\text { (lesiones) } \\
\text { - No conformidades con las normas legales } \\
\text { - Descompostura total del producto con posterior daño }\end{array}$ & 10 & $\begin{array}{l}\text { Ratio de falla muy alto } \\
\text { Fallos ocurren a gran escala } \\
(>100,000 \mathrm{ppm} 0>10 \%)\end{array}$ & 10 & $\begin{array}{l}\text { Falla será pasada a cliente sin ser detectada } \\
\text { Descubrimiento de la falla es improbable. La } \\
\text { fiabilidad de la detección no puede ser probada, } \\
\text { procedimientos de prueba inciertos }\end{array}$ & 10 \\
\hline $\begin{array}{l}\text { Efecto de falla alto } \\
\text { - Operatividad restringida del producto o partes } \\
\text { - Gran molestia de cliente } \\
\text { - Retrabajos o empleo de servicios }\end{array}$ & 8 & \begin{tabular}{|l} 
Ratio de falla alto \\
Fallos ocurren muy frecuentemente \\
$(<100,000$ ppm $0<10 \%)$
\end{tabular} & 8 & \begin{tabular}{|l|} 
Detección mínima \\
Descubrimiento de la falla es menos probable. La \\
fiabilidad de la detección probablemente no \\
puede ser probada
\end{tabular} & 8 \\
\hline \begin{tabular}{|l|} 
Efecto de falla moderado \\
- Leve deterioro del producto (perceptible por el cliente) \\
- Descontento del cliente \\
- Servicio al cliente
\end{tabular} & 5 & $\begin{array}{l}\text { Ratio de falla bajo } \\
\text { Fallos ocurren ocasionalmente } \\
(<10,000 \mathrm{ppm} 0<1 \%)\end{array}$ & 5 & \begin{tabular}{|l|} 
Detección baja \\
Falla es descubierta principalmente. Fiabilidad de \\
la detección puede ser probada, procedimientos \\
de prueba son relativamente certeros
\end{tabular} & 5 \\
\hline $\begin{array}{l}\text { Efecto de falla bajo } \\
\text { - Ligero deterioro óptico } \\
\text { - Ligera molestia del cliente } \\
\text { - Costos leves } \\
\end{array}$ & 3 & $\begin{array}{l}\text { Ratio de falla bajo } \\
\text { Fallos ocurren raramente }(<1,000 \\
\text { ppm } 0<0.1 \%)\end{array}$ & 3 & $\begin{array}{l}\text { Detección alta } \\
\text { Falla es descubierta con alta probabilidad. } \\
\text { Confirmado por varios métodos independientes }\end{array}$ & 3 \\
\hline $\begin{array}{l}\text { Efecto de falla no perceptible } \\
\text { - Deterioro de la función solo reconocible por el técnico } \\
\text { - Costos mínimos } \\
\text { - Deterioro ópticamente no percibido }\end{array}$ & 1 & $\begin{array}{l}\text { No hay acontecimientos } \\
\text { conocidos sobre productos } \\
\text { similares } \\
\text { Aproximadamente no ocurre fallas }\end{array}$ & 1 & $\begin{array}{l}\text { Detección muy alta } \\
\text { Definitivamente la falla es descubierta }\end{array}$ & 1 \\
\hline
\end{tabular}

Figura 3. Cuadro de valoraciones AMFE

Fuente. Bosch Group. Problem solving 


\subsection{Flujo de información del mercado}

Las atenciones por desperfectos en campo inician con las llamadas de los clientes a los servicios técnicos, los cuales se presentan a la dirección brindada por el cliente y se procede a solucionar el

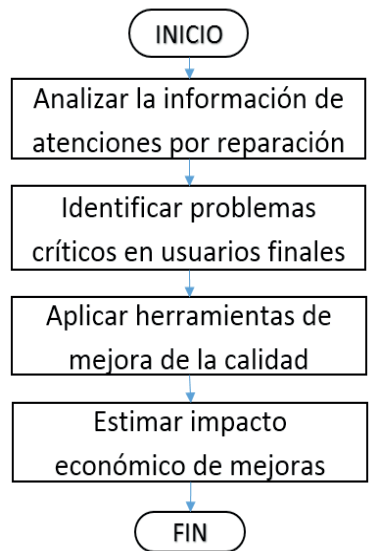

Figura 4. Diagrama de Flujo de Metodología Fuente. Elaboración propia

problema, ya sea in situ o en un taller, previa autorización del cliente. Al solucionar el desperfecto, los técnicos llenan una ficha que describe el trabajo realizado y el cliente procede a validar dicha información con su firma.

\section{ANÁLISIS Y APLICACIÓN DE HERRAMIEN- TAS DE CALIDAD}

\subsection{Análisis de atenciones por Departamento}

Las ventas se realizan en todo el Perú, por ello el análisis de la cantidad de atenciones se hará por departamento, siendo Lima quien representa poco más del $50 \%$ de las ventas totales (ver figura 5 ). Si se encuentra alguna falla en el producto, esta debe reflejarse en todos los departamentos del Perú en la misma proporción del volumen de ventas por departamento. Este análisis es crucial debido a que existe un conflicto de intereses al tener servicios técnicos que son terceros y que se benefician por cada atención.

\subsection{Análisis de atenciones por Modelo de Pro- ducto}

Los modelos de la línea en estudio se diferencian en volumen. Por esto es necesario un análisis ge-

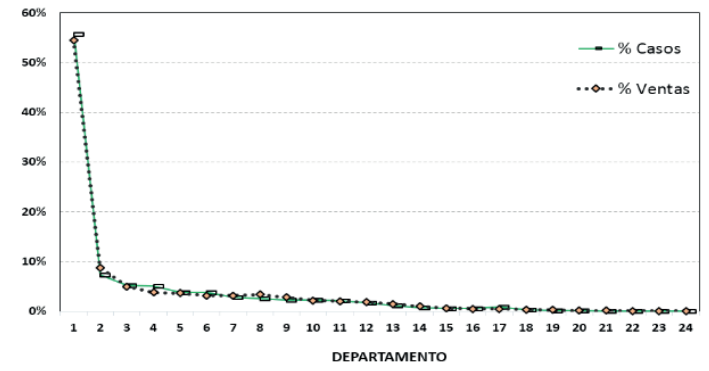

Figura 5. Nivel de atenciones Vs. Nivel de ventas por departamento

Fuente. Elaboración propia

neral del comportamiento de cada modelo del periodo de fabricación en estudio. Del mismo modo que el análisis anterior se hace un comparativo entre las ventas y el nivel de atenciones que se tiene por modelo de producto (ver figura 6).

A partir de los análisis anteriores, se puede concluir lo siguiente:

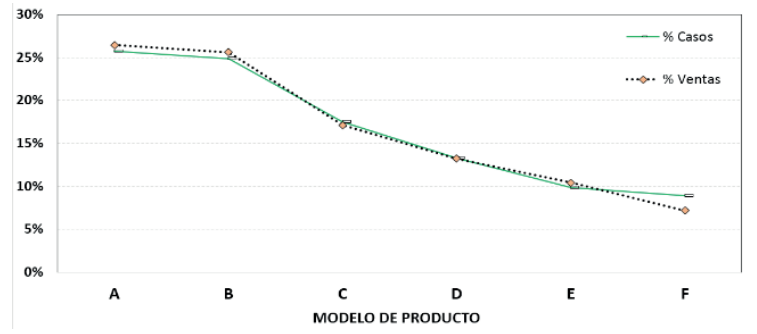

Figura 6. Nivel de atenciones Vs. Nivel de ventas por modelo Fuente. Elaboración propia

- No existen irregularidades en las atenciones de los servicios técnicos que sesguen el análisis de causas respecto al nivel de atenciones en los departamentos en que se venden los productos.

- Tampoco existe un modelo específico de producto de la línea en estudio con mayor nivel de atenciones en mercado. Las fallas se extienden de manera proporcional con su producción en todos los modelos.

Luego de este análisis previo para validar la información, se empezará a desagregar los tipos de fallo y cruzar la información disponible en mercado y fábrica para poder aplicar las herramientas de calidad de manera práctica. 


\subsection{Identificación de problemas críticos en usuarios finales}

El primer paso para analizar los problemas en las refrigeradoras que el usuario final percibe será identificar los principales síntomas de fallo (ver figura 7).

Seis síntomas representan más del $80 \%$ de fallos, se pueden agrupar en:

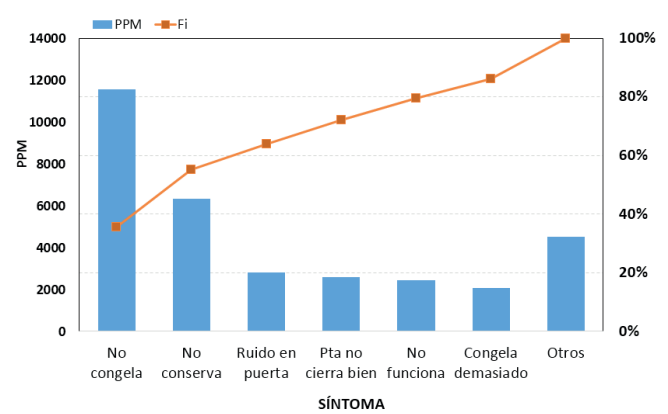

Figura 7. Pareto de síntomas de fallo en mercado - Línea No Frost

Fuente. Elaboración propia

- Fallos funcionales del sistema de refrigeración: No congela, No conserva, No funciona y Congela Demasiado. Este tipo de fallo está relacionado al funcionamiento de ciertos componentes que podrían ser el fusible térmico, timer, compresor, termostato, ventilador, evaporador o filtro entre los más probables.

- Fallos mecánicos: Ruido en puerta y Puerta no cierra bien. Pueden deberse al funcionamiento de la bocina, bisagra, puerta o burlete.

- Fallos estéticos: Dentro del grupo Otros debido a sus diversos síntomas y generalmente asociados a problemas de abolladuras en la puerta.

A continuación, en la figura 8 se presenta un Pareto de los componentes asignados por los técnicos que han generado más atenciones desde el período de fabricación 2014 a 2015.

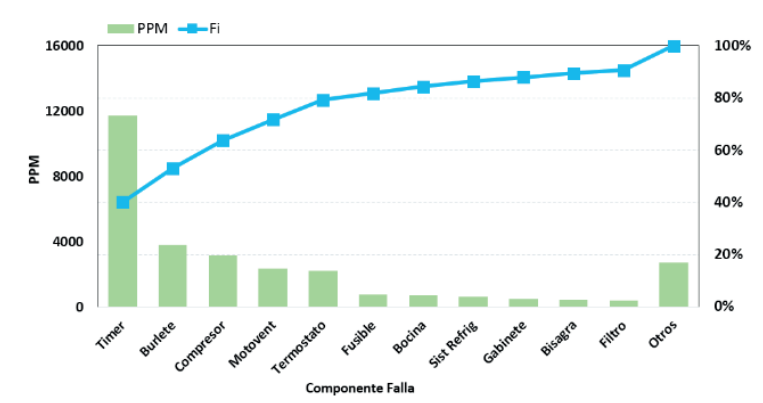

Figura 8. Pareto de las principales fallas en usuarios finales Fuente. Elaboración propia
Las figuras 7 y 8 muestran los principales problemas en mercado, algunos de estos ya tienen acciones en fábrica como los "problemas de ruido en puerta" que hasta el momento se nota la mejoría del problema. El síntoma de "puerta no cierra adecuadamente" se tiene identificado como un problema que deriva del mal funcionamiento del burlete, el cual es el segundo componente más cambiado por los técnicos y actualmente en proceso de mejora en fábrica. Los fallos estéticos tienen porcentajes de rechazo muy pequeño en mercado, por lo que se puede afirmar que los métodos usados para evitar la salida de estos defectos son eficaces.

En conclusión, se centra el estudio en proponer mejoras al grupo sintomático de fallos funcionales del sistema de refrigeración debido a que se ha venido realizando mejoras respecto a los grupos sintomáticos de fallos mecánicos $y$ fallos estéticos.

A continuación una breve descripción de los componentes con más atenciones y que pertenecen al grupo sintomático de fallos funcionales del sistema de refrigeración:

Timer: Componente que funciona eléctricamente y genera que el refrigerador descongele 25 minutos cada 6 horas para provocar un deshielo del congelador.

Compresor: Componente encargado de hacer circular el gas refrigerante por todo el sistema de refrigeración. Actualmente se utilizan 5 códigos de compresores en fábrica.

Motoventilador: Distribuye el frío desde congelador al conservador.

Termostato: Mide y controla las temperaturas del conservador y congelador. Este puede ser regulado por el usuario.

\subsection{Aplicación de herramientas de mejora de la calidad}

\section{Aplicación de la metodología 8D}

\section{PASO 1. Formar un equipo}

Los problemas principales se deben a fallos de componentes eléctricos que son importados, por lo tanto se convoca a los conocedores del funcionamiento eléctrico de las refrigeradoras: 
- Coordinador de servicios técnicos de todo el Perú.

- Dueño del proceso de ensamble de refrigeradoras.

- Encargado del control de Calidad de proveedores y de producto terminado.

\section{PASO 2. Definir el problema}

El problema se define claramente como: "Principales reparaciones en usuarios finales de refrigeradoras No Frost en garantía, producidas desde el 2014"

\section{PASO 3. Implementar acciones de contención}

El análisis y toma de acciones queda reducido a atacar la falla de los componentes eléctricos importados: Timer, Compresor, Ventilador y Termostato. Sin embargo, al estar en la etapa de contención, se debe establecer medidas que ayuden a reducir el riesgo de enviar productos finales a usuarios y que estos presenten el mismo o mayor nivel de falla ya conocido. El equipo propone:

- Debe ser aplicado el 100 por ciento de inspección para el Timer. Este componente representa un gran porcentaje en el nivel de falla total, por lo que debe ser tratado a manera de asegurar su reducción de fallo elevando su nivel de inspección y ser sometido a pruebas más exigentes.

- Informar el nivel de falla a proveedores y pedir soporte para la mejora del componente desde proveedor. Esto suponiendo que el nivel de falla alcanzado por cada componente se debe a la falla propia componente y no que sea la consecuencia de otras fallas.

- Reemplazo del compresor de la línea No frost por uno de mejor performance utilizado en las otras líneas. Se tiene en cuenta que esta acción encarece el producto, sin embargo es necesario el cambio por la cantidad de rechazos en mercado.

- Por lo expuesto, estas tres medidas de contención denotan acciones rápidas para reducir la tasa de fallos, sin embargo estas consumen recursos o encarecen los productos finales; este encarecimiento no es llevado al precio final, por lo que se traduce en reducción de ganancia. Estas medidas permanecerán hasta la búsqueda de la causa raíz y una solución robusta y permanente.

\section{PASO 4. Identificar y verificar la causa raíz}

\section{Análisis de información de mercado y de fábrica}

TIMER: Se estudia el nivel de fallo del componente en los últimos meses de producción y se obtiene lo siguiente:

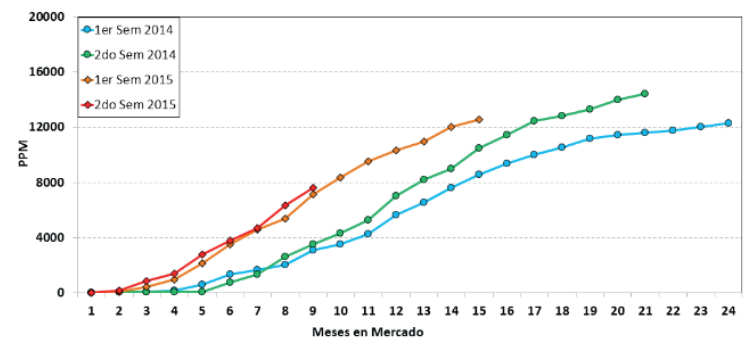

Figura 9. Indicador TCR del Timer por semestre de fabricación Fuente. Elaboración propia

En la figura 9 el indicador TCR de fábrica para este componente nos muestra que desde el 2014 las fallas del Timer se siguen presentando al mismo nivel antes de terminar los dos años en mercado. Aquí se descarta el hecho que gran parte de fallas se hayan centrado en períodos específicos de fabricación, ya que vemos su evolución de fallos por semestre, y estos alcanzan un nivel similar que es atribuible a la falla propia del componente.

COMPRESOR: Se hace un análisis del performance de cada Compresor que es utilizado en fábrica (ver figura 10):

Se puede ver claramente en la figura 10 que el mo-

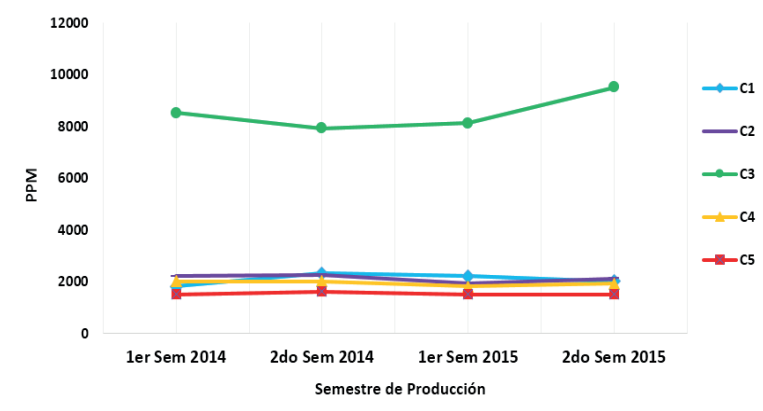

Figura 10. Indicador TCR de Compresores por semestre de fabricación

Fuente. Elaboración propia

delo $\mathrm{C} 3$ es el que tiene una tasa de fallos alto con respecto a los otros modelos. Justamente es este el modelo utilizado en la línea en estudio y que causa tantas atenciones en usuarios finales. Se debe tomar muy en cuenta que los cinco modelos utilizados en esta empresa son fabricados por el mismo proveedor y se ha venido trabajando con ellos infor- 
mando sobre el nivel de fallos del modelo de compresor C3, tanto en la fábrica peruana como otras fábricas de la corporación por más de 18 meses, no dando frutos positivos y encontrando siempre diversas causas.

MOTOVENTILADOR: Los análisis arrojan la siguiente distribución de tipos de fallo:

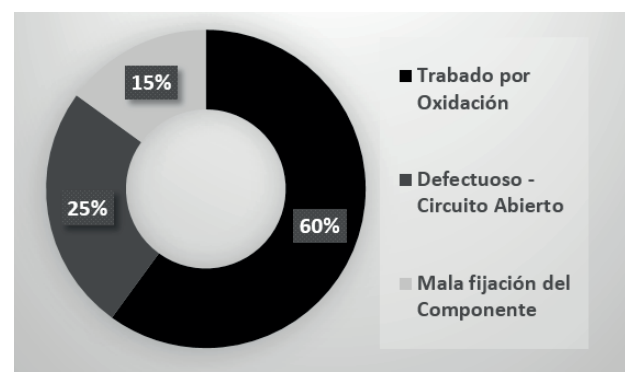

Figura 11. Distribución por tipo de fallo del motoventilador en mercado

Fuente. Elaboración propia

La distribución presentada en la figura 11 se pudo determinar debido a que la mayoría de los ventiladores que han sido cambiados por alguna falla en usuarios finales, son evaluados en la fábrica, teniendo así mayor evidencia del tipo de fallo. Se determina que el $60 \%$ de los problemas en el mercado se deben al trabamiento de este componente por oxidación. También con estas evaluaciones en fábrica se conoce que el $25 \%$ se debe a falla propia del componente por circuito abierto. Las atenciones que reportan algún fallo en el ventilador pero que no llegan a fábrica se deben a un ajuste que realiza el técnico debido a un mal montaje desde fábrica; este proceso se realiza manualmente e in situ.

TERMOSTATO: Las piezas falladas solicitadas de mercado son rápidamente enviadas a proveedor para el soporte respectivo debido a que es un proveedor corporativo, además de no contar con equipos adecuados para realizar las pruebas.

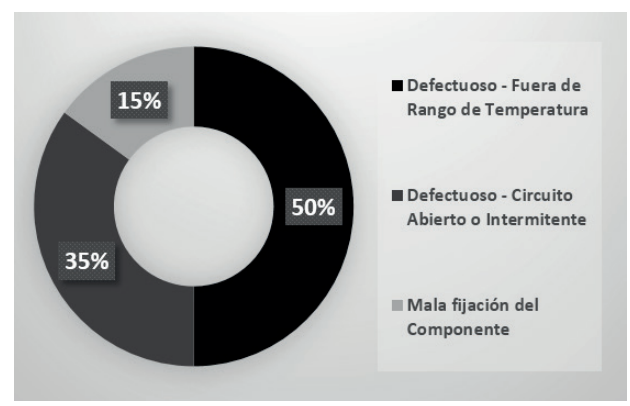

Figura12. Distribución por tipo de fallo del termostato en mercado

Fuente. Elaboración propia
La distribución de causas de fallo en mercado se presenta en la figura 12. El efecto del mal funcionamiento de este componente es crítico para el usuario y este abarca el $85 \%$ de los casos, por esto se iniciaron labores por parte del proveedor para mejorar la calidad de este componente.

\section{PASO 5. Determinar acciones correctivas per- manentes}

MOTOVENTILADOR: Se buscará reducir la tasa de fallo por medio de un recubrimiento que permita trabajar en óptimas condiciones a este componente, a pesar del incremento de costos. Se determina esta acción porque la mayor parte del fallo se debe a problemas de oxidación, esto debido a que este componente carece de un recubrimiento que impida o retrase esta oxidación que genera trabamiento.

COMPRESOR: Se verificó que el código usado en la línea en estudio tiene una alta tasa de fallos respecto a los otros cuatro utilizados en las otras líneas, por lo que se sugiere una estandarización de un modelo de Compresor, esto influye en los costos de manera negativa pero se buscará la compensación con el mayor volumen solicitado; así habrá una reducción de costos por economía de escala.

TIMER Y TERMOSTATO: Debido a una tasa alta de fallos de los componentes eléctricos, que como se ha mostrado, representan gran parte de los problemas ocurridos en clientes finales, el equipo propone un reemplazo de dos de los cuatro componentes. El Timer y Termostato serán reemplazados por un electrónico básico que tendrá como fin reducir la tasa de fallos de estos dos componentes en conjunto. Esta medida logrará concentrar los esfuerzos del equipo en un solo componente más complejo. Además, esta medida se propone debido a que se tiene información corporativa que sirve de soporte para poder lograr el cambio a un mediano plazo y con la confianza del buen desempeño de este componente, ya que es utilizado en otras fábricas de la corporación.

Por lo explicado, es necesario hacer un análisis que sea de implementación temprana en fábrica pero principalmente que asegure un mejor performance en el mercado. Con base en lo explicado y la información corporativa que se tiene del componente, es necesario utilizar el AMFE de diseño para poder prever los cambios necesarios y potenciales riesgos que afectarían al desempeño del producto final (ver tabla 1).

Como se observa, los puntos de mayor riesgo en el AMFE tienen actividades sugeridas, las cuales ya tienen responsables y fechas de implementación. 
Con este análisis previo del cambio, se tiene mayor seguridad de reducir las fallas en el producto terminado.

\section{PASO 6: Implementar y verificar las acciones correctivas permanentes}

Debido a que se requiere un cambio de diseño y reemplazo de componentes eléctricos, el área de desarrollo será el encargado de la implementación de la mayoría de los cambios. Además, estos cambios serán debidamente probados en la línea de ensamble previo al cambio permanente. En la tabla 2 se muestra un cronograma general de actividades por cada componente crítico en mercado.

\section{PASO 7: Prevenir la re-ocurrencia del problema y/o su causa raíz}

Los problemas encontrados en el mercado no fueron detectados en fábrica, por esto es necesario mantener un riguroso seguimiento de los principales problemas que encuentran los servicios técnicos por desperfectos en el producto. Desde el punto de vista del equipo es necesario tomar muestras que aceleren los fallos simulando las condiciones de uso. Las fallas presentadas en estas simulaciones podrán ser corregidas lo más pronto posible en fábrica. Para esto existen pruebas normadas en aparatos de refrigeración como los test de vida y las pruebas del peor escenario. Para las evaluacio-

Tabla 1. Aplicación del AMFE para la prevención de fallas en el producto

\begin{tabular}{|c|c|c|c|c|c|c|c|c|c|c|c|c|}
\hline $\begin{array}{c}\text { Interface I } \\
\text { Parte I } \\
\text { Proceso }\end{array}$ & Función & $\begin{array}{l}\text { Potencial } \\
\text { Efecto de } \\
\text { Falla }\end{array}$ & 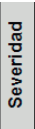 & $\begin{array}{c}\text { Potencial } \\
\text { Modo de Falla }\end{array}$ & $\begin{array}{c}\text { Potencial } \\
\text { Causa de Falla }\end{array}$ & 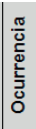 & 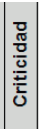 & $\begin{array}{l}\text { Modo de } \\
\text { Detección }\end{array}$ & 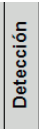 & RPN & $\begin{array}{l}\text { Acciones } \\
\text { sugeridas }\end{array}$ & 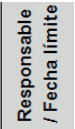 \\
\hline $\begin{array}{c}\text { EPS } \\
\text { superior } / \\
\text { inferior }\end{array}$ & \begin{tabular}{|c|} 
Permite la \\
distribución del aire \\
entre Freezer y \\
Conservador \\
\end{tabular} & $\begin{array}{c}\text { No permite } \\
\text { distribuir el aire }\end{array}$ & 8 & $\begin{array}{c}\text { No se forma el canal } \\
\text { y/o no permite el } \\
\text { correcto montaje del } \\
\text { damper }\end{array}$ & Mal diseño & 8 & A & $\begin{array}{c}\text { Se detecta en linea } \\
\text { de ensamble, no } \\
\text { permitiria ensamblar }\end{array}$ & 1 & 64 & $\begin{array}{c}\text { Fabricación de } \\
\text { prototipos con las } \\
\text { piezas }\end{array}$ & $\begin{array}{l}\text { Juan M. I } \\
\text { CW 45 }\end{array}$ \\
\hline $\begin{array}{c}\text { EPS } \\
\text { superior } I \\
\text { inferior }\end{array}$ & \begin{tabular}{|c|} 
Permite la \\
distribución del aire \\
entre Freezer y \\
Conservador \\
\end{tabular} & $\begin{array}{c}\text { No permite } \\
\text { distribuir el aire }\end{array}$ & 8 & \begin{tabular}{|c|}
$\begin{array}{c}\text { No se forma el canal } \\
\text { y/o no permite el } \\
\text { correcto montaje del } \\
\text { damper }\end{array}$ \\
\end{tabular} & \begin{tabular}{|c|}
$\begin{array}{c}\text { Densidad y geometria } \\
\text { inadecuada del } \\
\text { tecknoport (no cumple } \\
\text { especificaciones del } \\
\text { plano) }\end{array}$ \\
\end{tabular} & 3 & B & $\begin{array}{c}\text { Se detecta en linea } \\
\text { de ensamble, no } \\
\text { permitiria ensamblar }\end{array}$ & 1 & 24 & \begin{tabular}{|c|} 
Definir los \\
parametros de control \\
de la densidad del \\
material y geometria
\end{tabular} & $\begin{array}{l}\text { Frank M. } \\
\text { / CW 45 }\end{array}$ \\
\hline $\begin{array}{l}\text { EPS } \\
\text { superior }\end{array}$ & Aisla el aire helado & $\begin{array}{l}\text { No aisla aire } \\
\text { helado }\end{array}$ & 5 & $\begin{array}{l}\text { Condensación en } \\
\text { Conservador }\end{array}$ & $\begin{array}{c}\text { Densidad inadecuada } \\
\text { del tecknopor }\end{array}$ & 3 & B & $\begin{array}{c}\text { Se muestrea } \\
\text { semanalmente el } \\
\text { componente }\end{array}$ & 3 & 45 & - & - \\
\hline Damper & $\begin{array}{l}\text { Control de } \\
\text { temperatura }\end{array}$ & $\begin{array}{l}\text { No controla la } \\
\text { temperatura } \\
\text { entre } 0 \text { y } 6^{a} \mathrm{C} \\
\end{array}$ & 8 & \begin{tabular}{|c|}
$\begin{array}{c}\text { Temperaturas altas o } \\
\text { muy frias por flap } \\
\text { trabado }\end{array}$ \\
\end{tabular} & Falla en componente & 1 & c & $\begin{array}{l}\text { Muestras en } \\
\text { laboratorio }\end{array}$ & 5 & 40 & - & - \\
\hline Damper & $\begin{array}{l}\text { Control de } \\
\text { temperatura }\end{array}$ & $\begin{array}{l}\text { No controla la } \\
\text { temperatura } \\
\text { entre } 0 \text { y } 6^{a} \mathrm{C}\end{array}$ & 8 & \begin{tabular}{c|} 
Temperaturas altas o \\
muy frias en \\
conservador o \\
conaelador
\end{tabular} & $\mid \begin{array}{c}\text { Calibración del damper } \\
\text { en fábrica no } \\
\text { contempla todas las } \\
\text { condiciones de trabaio }\end{array}$ & 5 & A & $\begin{array}{l}\text { Muestras en } \\
\text { laboratorio }\end{array}$ & 8 & 320 & \begin{tabular}{|c|} 
Reevaluación de los \\
parámetros de trabajo \\
del sistema de \\
refriqeración
\end{tabular} & $\begin{array}{l}\text { Juan M. I } \\
\text { CW 45 }\end{array}$ \\
\hline Perilla & $\begin{array}{c}\text { Permite la } \\
\text { manipulación del } \\
\text { vastago del damper }\end{array}$ & $\begin{array}{c}\text { No se puede } \\
\text { manipular el } \\
\text { vástago del } \\
\text { damper }\end{array}$ & 5 & $\begin{array}{l}\text { No controla la } \\
\text { temperatura }\end{array}$ & $\begin{array}{c}\text { No se ajusta el vástago } \\
\text { y no se produce el giro- } \\
\text { a pesar de girar la } \\
\text { perilla (Diseño) }\end{array}$ & 8 & A & $\begin{array}{c}\text { Se detecta en } \\
\text { auditoria de producto } \\
\text { terminado }\end{array}$ & 3 & 120 & $\begin{array}{c}\text { Revisión de diseño } \\
\text { por Marketing en Pre- } \\
\text { piloto }\end{array}$ & $\begin{array}{l}\text { Juan M. I } \\
\text { CW 45 }\end{array}$ \\
\hline Perilla & \begin{tabular}{c|c|} 
Permite la \\
manipulación del \\
vastago del damper
\end{tabular} & $\begin{array}{c}\text { No se puede } \\
\text { manipular el } \\
\text { vástago del } \\
\text { damper }\end{array}$ & 5 & $\begin{array}{l}\text { No controla la } \\
\text { temperatura }\end{array}$ & \begin{tabular}{|c|} 
No se ajusta el vástago \\
y no se produce el giro- \\
a pesar de girar la \\
perilla (Inyección)
\end{tabular} & 1 & c & $\begin{array}{c}\text { Se detecta en } \\
\text { auditoria de producto } \\
\text { terminado }\end{array}$ & 3 & 15 & - & - \\
\hline Perilla & $\begin{array}{c}\text { Permite la } \\
\text { manipulación del } \\
\text { vastago del damper }\end{array}$ & $\begin{array}{l}\text { No se puede } \\
\text { manipular el } \\
\text { vástago del } \\
\text { damper }\end{array}$ & 5 & $\begin{array}{l}\text { No controla la } \\
\text { temperatura }\end{array}$ & $\begin{array}{c}\text { No se ajusta el vástago } \\
\text { y no se produce el giro- } \\
\text { a pesar de girar la } \\
\text { perilla (Montaje) }\end{array}$ & 3 & B & $\begin{array}{c}\text { Se detecta en } \\
\text { auditoria de producto } \\
\text { terminado }\end{array}$ & 3 & 45 & $\begin{array}{l}\text { Instructivo de montaje } \\
\text { en linea de ensamble }\end{array}$ & Eddy $S$. \\
\hline Perilla & $\begin{array}{c}\text { Perilla indica el nivel } \\
\text { de regulación }\end{array}$ & $\begin{array}{l}\text { No indica el } \\
\text { nivel de } \\
\text { regulación }\end{array}$ & 5 & $\begin{array}{l}\text { No controla la } \\
\text { temperatura }\end{array}$ & $\begin{array}{l}\text { Mala serigrafia, mal } \\
\text { acabado }\end{array}$ & 1 & c & $\begin{array}{l}\text { Se detecta } \\
\text { de ensar }\end{array}$ & 1 & 5 & 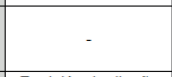 & - \\
\hline Perilla & $\begin{array}{c}\text { Perilla indica el nivel } \\
\text { de regulación }\end{array}$ & $\begin{array}{l}\text { No indica el } \\
\text { nivel de } \\
\text { requlación }\end{array}$ & 5 & $\begin{array}{l}\text { No controla la } \\
\text { temperatura }\end{array}$ & $\begin{array}{l}\text { No contar con } \\
\text { señalización }\end{array}$ & 5 & A & $\begin{array}{r}\text { Se detecta } \\
\text { de ensa }\end{array}$ & 1 & 25 & $\begin{array}{c}\text { Revisión de diseño } \\
\text { por Marketing en Pre- } \\
\text { piloto }\end{array}$ & $\begin{array}{l}\text { Juan M. I } \\
\text { CW 45 }\end{array}$ \\
\hline Perilla & \begin{tabular}{|c|}
$\begin{array}{c}\text { Perilla indica el nivel } \\
\text { de regulación }\end{array}$ \\
a
\end{tabular} & $\begin{array}{l}\text { No indica el } \\
\text { nivel de } \\
\text { regulación }\end{array}$ & 5 & $\begin{array}{l}\text { No controla la } \\
\text { temperatura }\end{array}$ & $\begin{array}{c}\text { No contar con acceso } \\
\text { visual de las } \\
\text { indicaciones }\end{array}$ & 3 & B & $\mid \begin{array}{c}\text { Se detecta en línea } \\
\text { de ensamble y } \\
\text { auditoria de producto } \\
\text { terminado }\end{array}$ & 1 & 15 & $\begin{array}{c}\text { Revisión de diseño } \\
\text { por Marketing en Pre- } \\
\text { piloto }\end{array}$ & $\begin{array}{l}\text { Juan M. I } \\
\text { CW } 45\end{array}$ \\
\hline Perilla & $\begin{array}{c}\text { Permite regulación } \\
\text { frecuente del usuario }\end{array}$ & $\begin{array}{c}\begin{array}{c}\text { No soporta una } \\
\text { frecuencia de } \\
\text { uso diario }\end{array} \\
\end{array}$ & 5 & $\begin{array}{l}\text { No controla la } \\
\text { temperatura }\end{array}$ & Muy debil (mal diseño) & 5 & A & & 8 & 200 & $\begin{array}{l}\text { Seguimiento de } \\
\text { defecto en Campo }\end{array}$ & Rick P. \\
\hline $\begin{array}{c}\text { EPS } \\
\text { Inferior }\end{array}$ & \begin{tabular}{|c|} 
Permite alojar la \\
perilla con el vástago \\
del dámper
\end{tabular} & \begin{tabular}{|c} 
No permite \\
alojar la perilla \\
con el vástago \\
del damper
\end{tabular} & 8 & \begin{tabular}{|c|} 
No se podria \\
ensamblar con el \\
damper y controlar la \\
temperatura
\end{tabular} & $\begin{array}{l}\text { Mala inyección del } \\
\text { agujero de pase de } \\
\text { perilla }\end{array}$ & 3 & B & \begin{tabular}{|c|} 
Se detecta en linea \\
de ensamble y \\
auditoria de producto \\
terminado
\end{tabular} & 1 & 24 & $\begin{array}{l}\text { Se evaluará puntos } \\
\text { críticos en pilotos }\end{array}$ & Eddy $S$. \\
\hline $\begin{array}{c}\text { EPS } \\
\text { Inferior }\end{array}$ & \begin{tabular}{|c|} 
Permite alojar la \\
perilla con el vástago \\
del dámper
\end{tabular} & $\begin{array}{c}\text { No permite } \\
\text { alojar la perilla } \\
\text { con el vástago } \\
\text { del damper }\end{array}$ & 8 & \begin{tabular}{|c|} 
No se podria \\
ensamblar con el \\
damper y controlar la \\
temperatura \\
\end{tabular} & $\begin{array}{l}\text { Agujero desalineado } \\
\text { por causa del molde }\end{array}$ & 3 & B & \begin{tabular}{|c|} 
Se detecta en línea \\
de ensamble y \\
auditoria de producto \\
terminado \\
\end{tabular} & 1 & 24 & $\begin{array}{l}\text { Se evaluará puntos } \\
\text { críticos en pilotos }\end{array}$ & Eddy S. \\
\hline $\begin{array}{c}\text { EPS } \\
\text { Inferior }\end{array}$ & $\left|\begin{array}{c}\text { Evitar la fuga de aire } \\
\text { helado por el orificio } \\
\text { donde pasa el } \\
\text { vástago del Damper }\end{array}\right|$ & $\begin{array}{c}\text { No evita la fuga } \\
\text { del aire helado } \\
\text { a traves del } \\
\text { orificio de la } \\
\text { perilla }\end{array}$ & 5 & $\begin{array}{l}\text { Muy bajas } \\
\text { temperaturas en } \\
\text { conservador }\end{array}$ & $\begin{array}{l}\text { Mal sellado entre el } \\
\text { EPS y el damper } \\
\text { (diseño) }\end{array}$ & 8 & A & $\begin{array}{c}\text { Se detecta en linea } \\
\text { de ensamble y } \\
\text { auditoria de producto } \\
\text { terminado }\end{array}$ & 1 & 40 & $\begin{array}{c}\text { Consideración en } \\
\text { prueba de } \\
\text { funcionamiento de } \\
\text { pilotos }\end{array}$ & $\begin{array}{l}\text { Alberto } \\
\text { M. }\end{array}$ \\
\hline
\end{tabular}

Fuente. Elaboración Propia 
Tabla 2. Cronograma general de actividades - Mejora de los principales defectos en mercado

\begin{tabular}{|c|c|c|c|c|c|c|c|c|c|c|c|c|c|c|c|}
\hline $\begin{array}{l}\text { Inicia: } \\
\text { Finaliza: }\end{array}$ & $\begin{array}{l}\text { Semana } 1 \\
\text { Semana } 16\end{array}$ & & \multicolumn{12}{|c|}{ Semana } & \\
\hline Respons. & \begin{tabular}{|c|} 
Componente \\
Crítico
\end{tabular} & Actividad & 1 & 23 & 45 & 6 & & 9 & 10 & 11 & 12 & 13 & 14 & 15 & 16 \\
\hline Juan M & Timer & $\begin{array}{l}\text { Desarrollo del Proyecto de } \\
\text { Electrónico Básico }\end{array}$ & & & & & & & & & & & & & \\
\hline Juan M & Termostato & $\begin{array}{l}\text { Desarrollo del Proyecto de } \\
\text { Electrónico Básico }\end{array}$ & & & & & & & & & & & & & \\
\hline Juan M & Compresor & $\begin{array}{l}\text { Reemplazo por otro modelo } \\
\text { de mejor performance en } \\
\text { mercado }\end{array}$ & & & & & & & & & & & & & \\
\hline José M & Motovent. & $\begin{array}{l}\text { Trabajo con Proveedor, } \\
\text { feedback para mejora de } \\
\text { componente por fallas } \\
\text { eléctricas }\end{array}$ & & & & & & & & & & & & & \\
\hline José M & Motovent. & $\begin{array}{l}\text { Recubrimiento en bobina de } \\
\text { motor para evitar corrosión y } \\
\text { trabamiento }\end{array}$ & & & & & & & & & & & & & \\
\hline
\end{tabular}

Fuente. Empresa en estudio

nes que permitan replicar lo que se presentaría en condiciones de uso normal es vital la información histórica de fallos de los productos.

\section{PASO 8: Reconocer los esfuerzos del equipo}

Se documentan los análisis y acciones del equipo de trabajo para eventos posteriores. El líder del $8 \mathrm{D}$ reconoce los esfuerzos del equipo y se da por concluido el objetivo del equipo.

\subsection{Estimación del impacto económico de las acciones propuestas}

Las mejoras propuestas apuntan a una reducción de fallos del producto en usuarios finales; teniendo como consecuencia ahorro de costos de garantía. Estos costos de atención dependen principalmente de la complejidad en la solución del fallo. Debido a que se tiene un estado inicial de las cantidades de atenciones por mes y por tipo de fallo, en la tabla 3 se obtiene un estimado del ahorro de costos de garantía al implementar las mejoras descritas en el 8D.

Tabla 3. Estimación del impacto económico

\begin{tabular}{|c|c|c|c|c|c|c|c|}
\hline Componente & Actividad & $\begin{array}{l}\text { Estado } \\
\text { actual } \\
\text { (PPMM) }\end{array}$ & $\begin{array}{l}\text { Costo de } \\
\text { Atención } \\
\text { Actual } \\
\text { (Dotares) }\end{array}$ & $\begin{array}{c}\text { Estado } \\
\text { Provectado } \\
\text { (PPM) }\end{array}$ & $\begin{array}{l}\text { Costo de } \\
\text { Atención } \\
\text { Proyectado } \\
\text { (Dólares) }\end{array}$ & $\begin{array}{l}\% \text { costo } \\
\text { Total por } \\
\text { Falla } \\
\text { (Actual) }\end{array}$ & 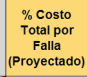 \\
\hline \begin{tabular}{c|} 
Timer + \\
Termostato
\end{tabular} & $\begin{array}{l}\text { Desarrollo del Proyecto } \\
\text { de Electrónico Básico }\end{array}$ & 17000 & 35 & 5000 & 50 & $40 \%$ & $17 \%$ \\
\hline Compresor & $\begin{array}{l}\text { Reemplazo por otro } \\
\text { modelo de mejor } \\
\text { performance en mercado }\end{array}$ & 4000 & 130 & 1800 & 130 & $25 \%$ & $11 \%$ \\
\hline Motovent. & \begin{tabular}{|l|} 
Kaizen con Proveedor: \\
mejora de componente \\
por fallas eléctricas. \\
Recubrimiento de bobina: \\
para evitar corrosión y \\
trabamiento
\end{tabular} & 2500 & 30 & 1000 & 30 & $6 \%$ & $2 \%$ \\
\hline
\end{tabular}

Fuente. Elaboración Propia
Es así que se estima una mejora total en costos de un $40 \%$ al término de la aplicación de todas las actividades propuestas. Por lo que se encuentra desde ya un sustento fuerte para empujar las acciones.

\section{CONCLUSIONES Y RECOMENDACIONES}

\subsection{Conclusiones}

- El análisis y solución de fallos de los productos en mercado le da a las empresas una ventaja competitiva que a largo plazo se verá reflejado en incremento de ventas, ya que se reducirán los fallos y se mejorará el porcentaje de clientes satisfechos.

- La aplicación de las herramientas de Calidad para identificar, corregir y prevenir futuros fallos establece bases fuertes en el sistema de calidad en la empresa, asegurando así un mejor desempeño de los productos en mercado.

- Es importante que las acciones correctivas sugeridas desde el equipo de trabajo al usar las herramientas de calidad sean debidamente probadas y aceptadas dentro del marco que establezca el mismo equipo. De no conseguir resultados cien por ciento satisfactorios; el análisis de riesgos determinará la aceptación de la acción correctiva o su rechazo. 


\subsection{RECOMENDACIONES}

- Una mayor preocupación de la Gerencia General en capacitaciones del personal donde se fomente la ideología de usar herramientas de Calidad para solucionar problemas con los artefactos producidos se traducirá en reducción de costos y mejora continua, tal como se presentó en este trabajo.

- Debido a que se estima una reducción del costo de atenciones en un $40 \%$ con las acciones sugeridas. Se propone un re-direccionamiento de dichos costos reducidos, ya que se podría emplear a los servicios técnicos para la instalación de los productos y así conseguir mayor satisfacción del cliente hasta llegar a su fidelización. Esta acción ya tiene un precedente en otra línea de la empresa.

\section{REFERENCIAS BIBLIOGRÁFICAS}

[1] Aldana de Vega, L. y Vargas Quiñones, M., (2011). Calidad y Servicio: Conceptos y Herramientas, Bogotá, Colombia: Eco Ediciones

[2] Bosch Group. (Mayo, 2013). Problem Solving. Quality Management in the Bosch Group. Robert Bosch Booklet, 1, 60 .

[3] Méndez Gamboa, M., (2008). Análisis de Confiabilidad utilizando modelos de componentes genéricos y matrices de programación de faIlas (Tesis de Maestría en Ciencias). Recuperado de http:// www.cenidet.edu.mx/subaca/ web-elec/tesis_mc/206MC_mamg.pdf 